\title{
Calculation of Inversion Time (TI) value for Fat Suppression in the Modified Spin Echo Sequence Method.
}

\author{
${ }^{1}$ Sunil Kalagi \\ ${ }^{1}$ BioMedical Physics, School of Medical Sciences, University of Aberdeen, Foresterhill, Aberdeen, UK \\ sunilkalagi@gmail.com
}

\begin{abstract}
Fat suppression is commonly used in Magnetic Resonance Imaging (MRI) to suppress the signal from adipose tissue or detect adipose tissue. Due to short relaxation times, fat has a high signal on magnetic resonance images (MRI). This high signal, easily recognized on MRI. The high signal due to fat may be responsible for artifacts such as ghosting and chemical shift. Lastly, a contrast enhancing tumor may be hidden by the surrounding fat. These problems have prompted development of fat suppression techniques. in MRI. Fat may be suppressed on the basis of its difference in resonance frequency with water by means of frequency selective pulses or phase contrast techniques, or on the basis of its short $\mathrm{TI}$ relaxation time by means of inversion recovery sequences. The aim of this paper is to study the Fat suppression technique using Inversion recovery Pulse sequence by calculating $\mathrm{TI}_{\text {null }}$ parameter for the pulse sequence.
\end{abstract}

Keywords: Fat Suppression; Inversion Time; Spin Echo Sequence.

\section{Introduction}

In MRI fat suppression is essential for accurate diagnosis which may obscure underlying pathology. [1-6] The different chemical environments of fat and water cause fat to precess at a lower resonant frequency than water. At 4.7T fat precesses at about $700 \mathrm{~Hz}$ less than water.[7-8] This is usually expressed as -3.5 parts per million of the main field strength. [8-9]

A magnetic field gradient allows the resonant frequency of an object to be mapped to its position along the gradient direction [10-12]. Therefore differences in resonant frequency result in differences in position. This results in fat being shifted relative to water in an image and is called the chemical shift artefact $\delta .[13-15]$ This is described in terms of frequency as follows

$$
\delta=\left(\Delta f / f_{o}\right) 10^{6}
$$

where $\Delta f=$ change in frequency $(\mathrm{Hz})$ and $\mathrm{f}_{\mathrm{o}}=$ Centre frequency $(\mathrm{Hz})$. In images this shift is characterised by a dark edge between the fat and water [1,8 and 13].

In situations where the chemical shift artefact is present the fat signal can obscure the water signal from underlying tissue. Here the aim was to find the appropriate value of the inversion time (TI), for use in the modified spin echo sequence, which would suppress the contribution from the fat.[13-15] 
Inversion recovery pulse sequence: Inversion recovery (IR) is a conventional spin echo (SE) sequence preceded by a $180^{\circ}$ inverting pulse. In other words, if a SE sequence is denoted by $\left(90^{\circ}-180^{\circ}\right.$-echo), the IR sequence can be written as $180^{\circ}-\left(90^{\circ}-180^{\circ}-\right.$ echo).[1-8]

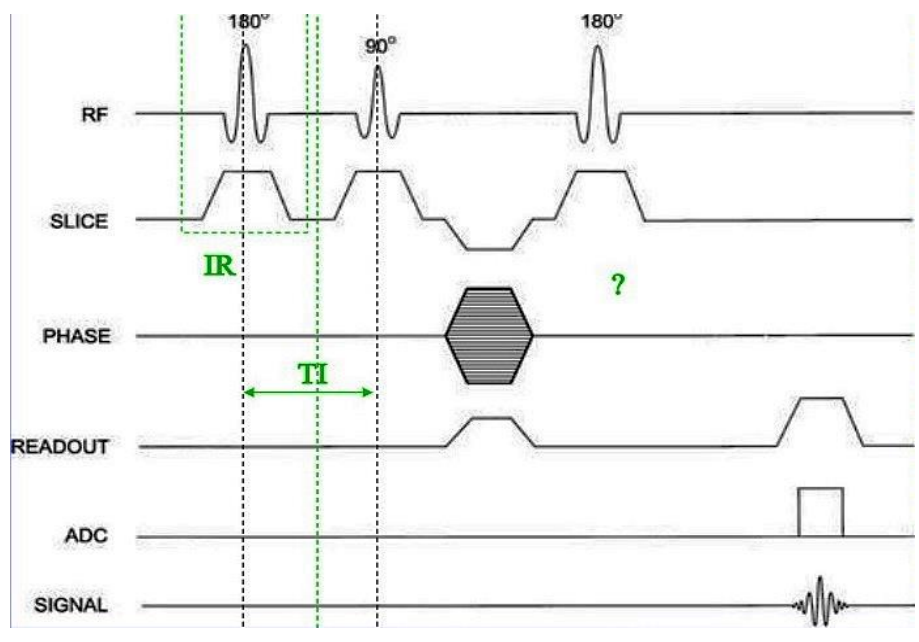

Figure 1. Inversion Recovery (IR) pulse sequence.

The time between the $180^{\circ}$ inverting pulse and the $90^{\circ}$ pulse is called the inversion time (TI). The repetition time (TR) and echo time (TE) are defined as they are for spin echo.[1-8]

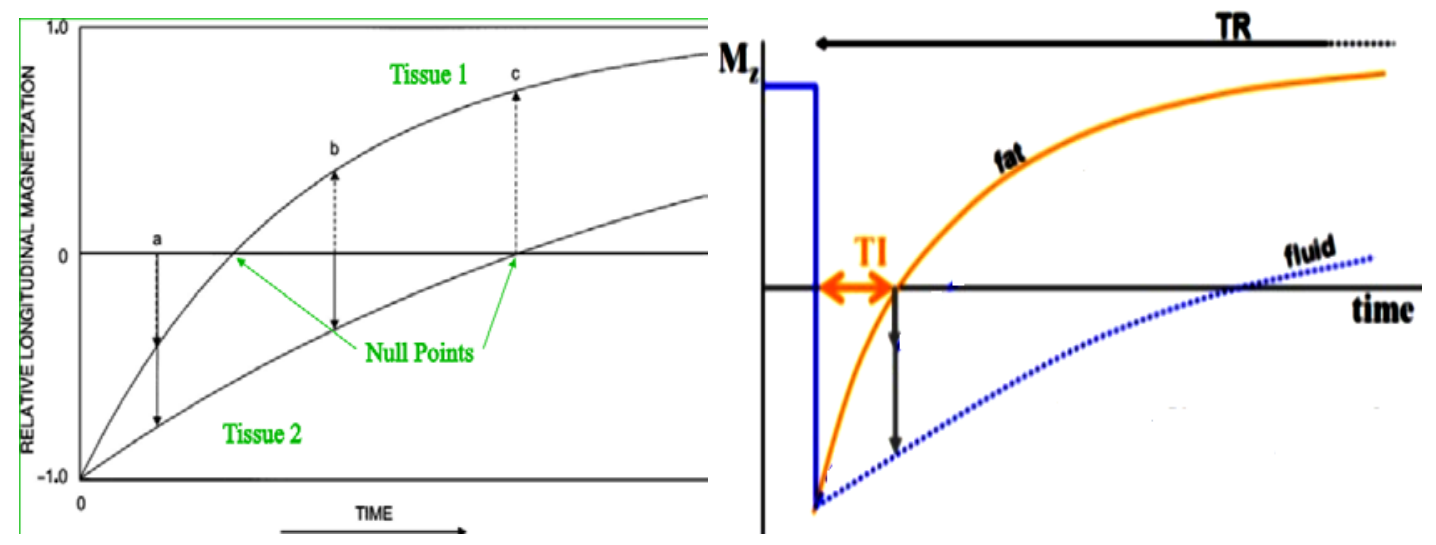

Figure 2 (a) Contrast of Fat(Tissue1) and Water (Tissue2)tissues (b) TI for fat suppression

\section{Method}

\subsection{MRI System:}

The Preclinical 4.7 Tesla micro MRI system(Surrey Medical Imaging Systems, UK) located at Biomedical physics department of University Aberdeen was used for our research project. The system consisted of: Magnex 4.7 Tesla superconducting magnet; MR Solutions console and pulse programmer; Magnex shielded gradient coils with Techron drivers capable of producing field gradients of $90 \mathrm{mT} / \mathrm{m}$ with rise times of $200 \mu \mathrm{s}$ and Morris Instruments birdcage transmit/receive coil, and other RF coils of in-house design. 


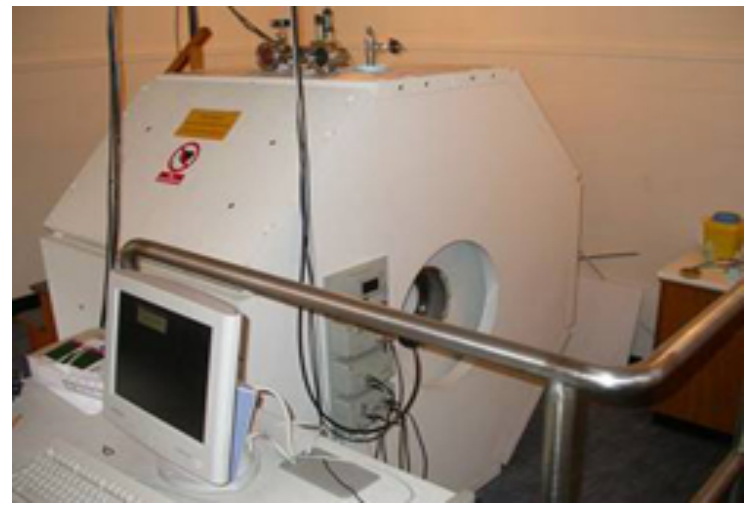

Figure 3. The Preclinical 4.7 Tesla micro MRI system with console at University of Aberdeen.

\subsection{Sample}

A set of 7 tubes containing either water or vegetable oil were imaged together. In order to reduce the relaxation time the water had been doped with copper sulphate. Without fat suppression images were made using the standard spin echo sequence. Two images were obtained in this way - one with an image bandwidth of $25 \mathrm{kHz}$ and the other with $12.5 \mathrm{kHz}$. The other parameters associated with these images are as follows: Field - of view $=100 \mathrm{~mm}$, Slice thickness $=5 \mathrm{~mm}$, Echo time $(T E)=40 \mathrm{~ms}$ and the time between $90^{\circ}$ pulses $(\mathrm{TR})=1000 \mathrm{~ms}$.

To achieve fat suppression it was necessary to employ an inversion recovery pulse sequence. This pulse sequence includes a $180^{\circ}$ pulse to invert the spins onto the negative $z-$ axis. Following this the magnetisation vector slowly returns up the $z-$ axis towards equilibrium. This is due to spin - lattice (T1) relaxation. When a $90^{\circ}$ pulse is applied before equilibrium is reached the magnetisation is transferred from the $z$ - axis to the $x-y$ plane. Spin - spin (T2) relaxation results in the free induction signal (FIS).

The time between the $180^{\circ}$ and $90^{\circ}$ pulses is the inversion time ( $\left.\mathrm{TI}\right)$. If $\mathrm{TI}$ coincides with the time at which the longitudinal magnetisation $M_{z}$ of the fat is zero then there is no magnetisation from fat to be transferred to the $x-y$ plane. Therefore there is zero contribution of the fat spins to the FIS. This value of $\mathrm{TI}$ is referred to as $\mathrm{TI}_{\mathrm{NULL}}$ and its relationship to $\mathrm{T}_{1}$ is given below. As the fat and water have different values of $\mathrm{T}_{1}$ the water will still contribute to the FIS. [1, 2, 3]

$$
\begin{aligned}
& \mathrm{M}_{\mathrm{z}}=\mathrm{M}_{\mathrm{o}}\left(1-2 \exp \left(-\mathrm{TI} / \mathrm{T}_{1}\right)\right)=0 \\
& \therefore \mathrm{TI}_{\mathrm{NULL}}=0.693 \mathrm{~T}_{1 \text { (water/fat })}
\end{aligned}
$$

To obtain an image in which the signals from the tubes of fat are suppressed this value of $T I_{\text {NuL }}$ for fat was used in a modified spin echo pulse sequence given below.

$$
\left[180^{\circ}-\mathrm{TI}-90^{\circ}-\mathrm{TE} / 2-180^{\circ}-\mathrm{TE} / 2-\text { signal }-\mathrm{TR}-\right]^{\mathrm{n}}
$$

This is the spin echo pulse sequence with an additional inversion pulse prior to the start of the spin echo sequence. Here $\mathrm{n}=256$.

The main field was generated by a 4.7T superconducting solenoid. The RF coil's impedance at resonance was matched to the standard $50 \Omega$ output/input impedance of RF power amplifiers/preamplifiers to 
ensure efficient transfer from the coil. Shimming was carried out to correct for inhomogeneities in the magnetic field.

\section{Results and Discussion}

\subsection{Without Fat Suppression}

The images obtained using the spin echo pulse sequence (without fat suppression) were reconstructed using a contrast factor of 0.05 and are shown below.

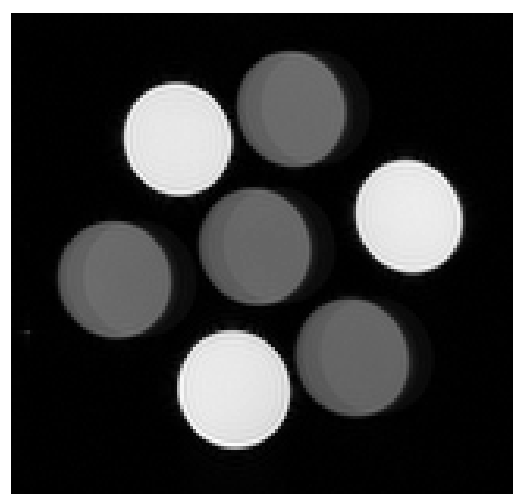

Figure 4. Spin echo image obtained using a bandwidth of $25 \mathrm{kHz}$

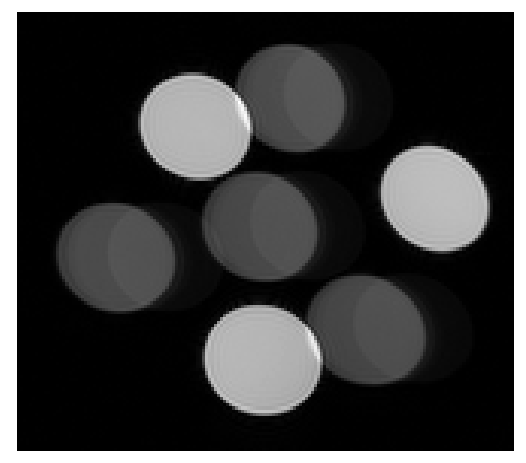

Figure 5. Spin echo image obtained using a bandwidth of $12.5 \mathrm{kHz}$

At higher field strengths $(>0.5 T)$ there is known to be a significant shift in fat relative to water . By comparing the images obtained at 25 and $12.5 \mathrm{kHz}$ it was therefore concluded that the bright circles correspond to water and the dark circles to fat. The images show that in addition to being shifted the dark circles are slightly distorted. Also there is a faint circle in figure 5 in the same position as the fat peak in figure 4. This corresponds to the fat also containing some water. The observed shifts of the fat in figure 5 are quantified in the following section.

\section{(1.1) Chemical shift artefact}

The shift of the fat peak in figure 5 relative to its position in the higher frequency image was found by measuring the distance from the left hand edge of the faint water circle still present in figure 5(position $\mathrm{x} 1$ ) to the left hand edge of the shifted fat circle (position $\times 2$ ) (see figure 6). 


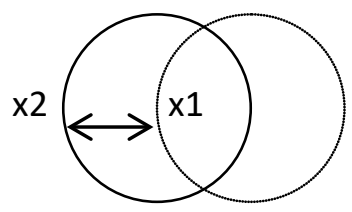

Figure 6. Diagram showing the positions on the fat circles of figure 5 used to calculate the shift of the fat in the $12.5 \mathrm{kHz}$ image relative to its position in the $25 \mathrm{kHz}$ image.

The values used to measure the shift of the 4 fat samples in figure 5 are as follows

Table 1. Values of positions on the fat circles in figure 5 used to calculate the shift $(\Delta x)$ of fat relative to water in millimetres $(\mathrm{mm})$.

\begin{tabular}{|c|c|c|}
\hline $\mathrm{x} 1(\mathrm{~mm})$ & $\mathrm{x} 2(\mathrm{~mm})$ & $\Delta \mathrm{x}(\mathrm{mm})$ \\
\hline-24.6 & -30.9 & -6.3 \\
\hline-6.3 & -12.1 & -5.8 \\
\hline-7.8 & -1.6 & -6.2 \\
\hline-1.6 & -7.8 & -6.2 \\
\hline
\end{tabular}

In figure 5 the bandwidth is $12.5 \mathrm{kHz}$ and the centre frequency $\left(\mathrm{f}_{\mathrm{o}}\right)$ is $199.9 \mathrm{MHz}$. Since the field of view is $100 \mathrm{~mm} \times 100 \mathrm{~mm}, 1 \mathrm{~mm}$ corresponds to $125 \mathrm{~Hz}$. This allows the shift in millimetres $(\Delta x)$ to be converted to a shift in frequency $(\Delta f)$. Using these values and the centre frequency $\left(f_{o}=199.1 \mathrm{MHz}\right)$ the chemical shift $(\delta)$ can be calculated according to equation 1 . These values are given in Table 2 and are close to the expected shift of fat relative to water (-3.5ppm) .

Table 2. chemical shift values $\delta$ corresponding to the fat samples in figure 5 calculated according to equation 1 using change in frequency $\Delta \mathrm{f}$.

(1.2) $\underline{T I}_{\underline{N U L L}}$ and $T_{1}$

\begin{tabular}{|c|c|c|}
\hline$\Delta x(\mathrm{~mm})$ & $\Delta \mathrm{f}(\mathrm{Hz})$ & $\delta(\mathrm{ppm})$ \\
\hline-6.3 & -787.5 & -3.94 \\
\hline-5.8 & -725 & -3.64 \\
\hline-6.2 & -775 & -3.89 \\
\hline-6.2 & -775 & -3.89 \\
\hline
\end{tabular}

Using the inversion recovery pulse sequence the $T I_{\text {NULL }}$ value was estimated by suppressing the fat peak of the Free Induction Signal (FIS). By trial and error this was found to be $140 \mathrm{~ms}$. From this $\mathrm{T}_{1}$ was calculated according to equation 3 and was found to be $201.6 \mathrm{~ms}$.

\subsection{With Fat Suppression}

The image below was obtained by including the estimated value of $\mathrm{TI}_{\mathrm{NuLL}}$ for fat (140ms) in the modified spin echo pulse sequence (see theory section). It was reconstructed using a contrast factor of 0.2 . 


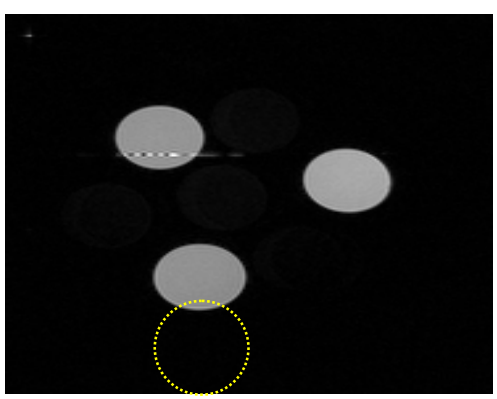

Figure 7. Fat suppression achieved using the modified spin echo sequence with TINuL and a bandwidth of 25 $\mathrm{kHz}$. The yellow circle represents the approximate region of the fat sample in figure 4.

\subsection{Quantifying Fat suppression}

\subsubsection{Visual inspection}

That only the brighter circles remain indicates that fat suppression has been successful. However the circles corresponding to the water samples look darker than in previous images. The line across the top left hand side water sample is an artefact caused by the imager.

\subsubsection{Fat to Water Ratio}

To formally quantify fat suppression it is necessary to compare the fat to water signal intensity with (figure 7) and without (figure 4) fat suppression. For this comparison the water sample at the bottom of figures 4 and 7 was used. The fat sample at the top of the figure 4 and the region defined by the yellow circle at the top of figure 7were also used. Taking the different scaling of the two images into account the mean pixel intensities of the regions of fat and water described above are given in the table below.

Table 3. A comparison of the mean pixel intensities of regions of fat and water in figures 4 and 7 (i.e. without and with fat suppression respectively).

\begin{tabular}{|c|c|}
\hline Regions & Mean pixel intensity \\
\hline $\begin{array}{c}\text { Water - figure1 } \\
\text { (fat unsuppressed) }\end{array}$ & 19544 \\
\hline $\begin{array}{c}\text { Fat - figure1 } \\
\text { (fat unsuppressed) }\end{array}$ & 9008 \\
\hline $\begin{array}{c}\text { Water - figure 4 } \\
\text { (Fat suppressed) }\end{array}$ & 4648 \\
\hline $\begin{array}{c}\text { Fat - figure 4 } \\
\text { (Fat suppressed) }\end{array}$ & 306 \\
\hline
\end{tabular}

From this table the (fat/water) ratio for the unsuppressed image (figure 4) was found to be 0.46 and that for the suppressed image (figure 7) was found to be 0.07 .

The unsuppressed to suppressed fat ratio was also calculated from the above values and was found to be $\sim 30$. The corresponding ratio for water in the different images was found to be $\sim 4$. Therefore fat suppression is $\sim 7$ times higher than water suppression between the two images. This shows that there has been considerable suppression of the fat using $\mathrm{TI}_{\mathrm{NULL}}=140 \mathrm{~ms}$ in the modified spin echo pulse sequence. 
Sunil Kalagi; Calculation of Inversion Time (TI) value for Fat Suppression in the Modified Spin Echo Sequence Method. Journal of Biomedical Engineering and Medical Imaging, Volume 5, No 2, April (2018), pp 21-28

\section{Conclusion}

Two images were obtained using the standard pulse echo sequence with bandwidths of $25 \mathrm{kHz}$ and 12.5 $\mathrm{kHz}$. As the dark circles were observed to shift relative to water for the lower frequency image they were identified as corresponding to the fat samples. From this image the shifts for the four fat samples were calculated as $-3.94,-3.63,-3.89$ and $-3.89 \mathrm{ppm}$. These values are all within $0.44 \mathrm{ppm}$ of the expected shift for this field strength.

By minimizing the contribution of the fat samples to the FIS, TINULL was found to be $140 \mathrm{~ms}$. This corresponds to a $T_{1}$ value of $201.6 \mathrm{~ms}$. This $T_{1}$ value is about 5 times shorter than TR and is about 5 times longer than TE.

Fat suppression was then attempted using the above value of $T I_{\text {NuLl. }}$ This was quantified by calculating the fat/water signal of the unsuppressed and suppressed images. These were found to be 0.46 and 0.07 respectively. This indicates that considerable suppression of the fat signals had been achieved. However some suppression of the water has also taken place. Selection of a fat suppression technique should depend on the purpose of the fat suppression(contrast enhancement vs tissue characterization) and the amount of fat in the tissue being studied.

\section{ACKNOWLEDGEMENTS}

I would like to thank my supervisor Dr. Hugh Seton for his assistance and suggestions throughout this project. Without his advice, enthusiasm and patience, this work would never have been achieved.

\section{REFERENCES}

[1] De Kerviler, E., et al. "Fat suppression techniques in MRI: an update." Biomedicine \& pharmacotherapy 52.2 (1998): 69-75.

[2] D. J. Blezek, W. T. Dixon and P. J. Dhawale, "Single image phase-based MRI fat suppression expectation maximization algorithm," 2005 IEEE Computer Society Conference on Computer Vision and Pattern Recognition (CVPR'05), 2005, pp. 540-546 vol. 2.

[3] Bley, T. A., Wieben, O., François, C. J., Brittain, J. H. and Reeder, S. B. (2010), Fat and water magnetic resonance imaging. J. Magn. Reson. Imaging, 31: 4-18. doi:10.1002/jmri.21895.

[4] Reeder, S. B., Yu, H., Johnson, J. W., Shimakawa, A., Brittain, J. H., Pelc, N. J., Beaulieu, C. F. and Gold, G. E. (2006), $T_{1}$ - and $T_{2}$-weighted fast spin-echo imaging of the brachial plexus and cervical spine with IDEAL water-fat separation. J. Magn. Reson. Imaging, 24: 825-832. doi:10.1002/jmri.20721

[5] Yu, H., Reeder, S. B., McKenzie, C. A., Brau, A. C. S., Shimakawa, A., Brittain, J. H. and Pelc, N. J. (2006), Single acquisition water-fat separation: Feasibility study for dynamic imaging. Magn. Reson. Med., 55: 413-422. doi:10.1002/mrm.20771

[6] Zhang, W., Goldhaber, D. M. and Kramer, D. M. (1996), Separation of water and fat MR images in a single scan at .35 T using „sandwich” echoes. J. Magn. Reson. Imaging, 6: 909-917. doi:10.1002/jmri.1880060612 
[7] Palosaari, Kari, et al. "Fat suppression gradient-echo magnetic resonance imaging of experimental articular cartilage lesions: Comparison between phase-contrast method at $0.23 \mathrm{~T}$ and chemical shift selective method at 1.5 T." Journal of Magnetic Resonance Imaging 18.2 (2003): 225-231.

[8] Xiang, Q.-S. and An, L. (1997), Water-fat imaging with direct phase encoding. J. Magn. Reson. Imaging, 7: 1002-1015. doi:10.1002/jmri.1880070612

[9] Ma, J., Vu, A. T., Son, J. B., Choi, H. and Hazle, J. D. (2006), Fat-suppressed three-dimensional dual echo dixon technique for contrast agent enhanced MRI. J. Magn. Reson. Imaging, 23: 36-41. doi:10.1002/jmri.20470

[10] Miller, T. T., et al. "Fat-suppressed MRI of musculoskeletal infection: fast T2-weighted techniques versus gadolinium-enhanced T1-weighted images." Skeletal radiology 26.11 (1997): 654-658.

[11] Reeder, Scott B., et al. "Quantitative assessment of liver fat with magnetic resonance imaging and spectroscopy." Journal of magnetic resonance imaging 34.4 (2011): 729-749.

[12] Mirowitz, ScotiA, et al. "MR imaging of bone marrow lesions: relative conspicuousness on T1-weighted, fat-suppressed T2-weighted, and STIR images." AJR. American journal of roentgenology 162.1 (1994): 215221.

[13] Lavdas, loannis, et al. "Stripline resonator and preamplifier for preclinical magnetic resonance imaging at 4.7 T." Magnetic Resonance Materials in Physics, Biology and Medicine 24.6 (2011): 331-337.

[14] Lin, Chen, Clark David Rogers, and Shadie Majidi. "Fat suppression techniques in breast magnetic resonance imaging: a critical comparison and state of the art." (2015).

[15] Tien, Robet Dt. "Fat-suppression MR imaging in neuroradiology: techniques and clinical application." AJR. American journal of roentgenology 158.2 (1992): 369-379. 